\title{
ArcheoSciences
}

Revue d'archéométrie

$30 \mid 2006$

Varia

\section{Dynamiques morpho-sédimentaires et peuplement sur le Rocher de Roquebrune-sur-Argens (Var)}

Morpho-sedimentary dynamics and settlement patterns on the Roquebrune-onArgens hill (southern France)

Mireille Provansal, Frédérique Bertoncello et Guillaume Gili

\section{CpenEdition}

Journals

Édition électronique

URL : https://journals.openedition.org/archeosciences/360

DOI : 10.4000/archeosciences.360

ISBN : 978-2-7535-1595-6

ISSN : 2104-3728

Éditeur

Presses universitaires de Rennes

Édition imprimée

Date de publication : 31 décembre 2006

Pagination : $173-180$

ISBN : 978-2-7535-0456-1

ISSN : 1960-1360

Référence électronique

Mireille Provansal, Frédérique Bertoncello et Guillaume Gili, « Dynamiques morpho-sédimentaires et peuplement sur le Rocher de Roquebrune-sur-Argens (Var) », ArcheoSciences [En ligne], 30 | 2006, document 17, mis en ligne le 31 décembre 2008, consulté le 01 février 2022. URL : http:// journals.openedition.org/archeosciences/360 ; DOI : https://doi.org/10.4000/archeosciences.360

Article L.111-1 du Code de la propriété intellectuelle. 


\title{
Dynamiques morpho-sédimentaires et peuplement sur le Rocher de Roquebrune-sur-Argens (Var)
}

\author{
Mireille Provansal*, Frédérique Bertoncello** et Guillaume Gili*
}

Résumé : Le Rocher de Roquebrune, dont l'occupation protohistorique et antique est bien connue, offre la possibilité de confronter les observations géomorphologiques et archéologiques afin d'appréhender, à l'échelle de ce petit massif, l'histoire des processus érosifs dans le bassin-versant de l'Argens. Initiée dans le cadre du programme Éclipse, l'étude géomorphologique de deux petits vallons du Rocher a mis en évidence une phase exceptionnelle de torrentialité à l'Âge du Fer, qui complète les nombreuses observations réalisées depuis 15 ans en Provence varoise. L'épisode climatique du Petit Âge Glaciaire, quoique représenté dans la stratigraphie, n’est pas associé à des impacts sédimentaires aussi spectaculaires. Les résultats montrent la complexité des interactions entre processus climatiques et anthropiques, dans lesquels entrent en jeu les modalités de l'occupation des sols, mais aussi les types de mise en valeur agricole, dont la réalité archéologique est bien plus difficile à percevoir.

\begin{abstract}
The Preroman and Roman settlements on the Rochers de Roquebrune are well know and give the possibility to be confronted to the geomorphic observations, in the aim to understand the story of the erosion at the scale of this little mountain, and, later, to the feeding of the deltaic progradation of the Argens River. Helped by the geo-archeologiaal programm Éclipse, the geomorphic study of two little valleys describes two different detritic pulses: the first, during the Iron Age, is contemporary of numerous observations of the same type on archeological sites in southern France; le second, corresponding to the Little Ice Age climatic phase, is not so important as usual. The results show the complexity of the interactions between the climatic and anthropic processes: the type of settlement pattern certainly play a role, as well as agricultural modes, although these are more difficult to perceive with the sole archeological remains.
\end{abstract}

Mots clés : Détritisme, anthropisation, Protohistoire, Antiquité, Petit Age Glaciaire, Provence orientale.

Key words: Detritism, anthropisation, protohistory, Antiquiyty, Little Ice Age, eastern Provence.

\section{Problématique de la Recherche}

Prenant la suite du projet de recherche "Caractériser la crise climatique des $\mathrm{VI}^{\mathrm{e}}-\mathrm{VII}^{\mathrm{e}}$ siècles en Provence dans ses relations avec la crise des environnements et des sociétés " coordonné par Ph. Leveau dans le cadre de l'appel d'offres "Éclipse I ", dont un volet concernait la région de Fréjus, le Programme Collectif de Recherches « Occupation du sol et patrimoine archéologique dans la basse vallée de l'Argens" a bénéficié de certaines opérations réalisées dans le cadre de ce projet. C'est le cas de l'étude géomorphologique effectuée au pied du Rocher de Roquebrune-sur-Argens en
2001 (Fig. 1a). Deux sites ont été étudiés de part et d'autre du Rocher : au nord, une coupe a été observée à l'aval du petit torrent de «La Roche de Palay » issu du Rocher et se jetant dans l'Argens en rive droite; sur le versant sud, une tranchée a été creusée en travers de la basse terrasse du vallon de Rabinon, au lieu-dit "Varaille» (Fig. 1b). En raison du temps nécessaire à l'analyse des échantillons prélevés et des délais d'obtention des datations radiocarbone, l'étude de ces sites et leur interprétation se sont non seulement inscrites dans le cadre temporel du PCR, mais se sont enrichies de la réflexion collective engagée sur l'ensemble de la basse vallée de l'Argens au sein de ce programme.

\footnotetext{
*UMR 6635 CEREGE Europôle de l'Arbois, BP 80, F-13545 Aix-en-Provence. (provansal@cerege.fr)

** CEPAM, UMR 6130, 250, rue Albert-Einstein, Sophia Antipolis, 06560 Valbonne. (fberton@cepam.cnrs.fr)
} 


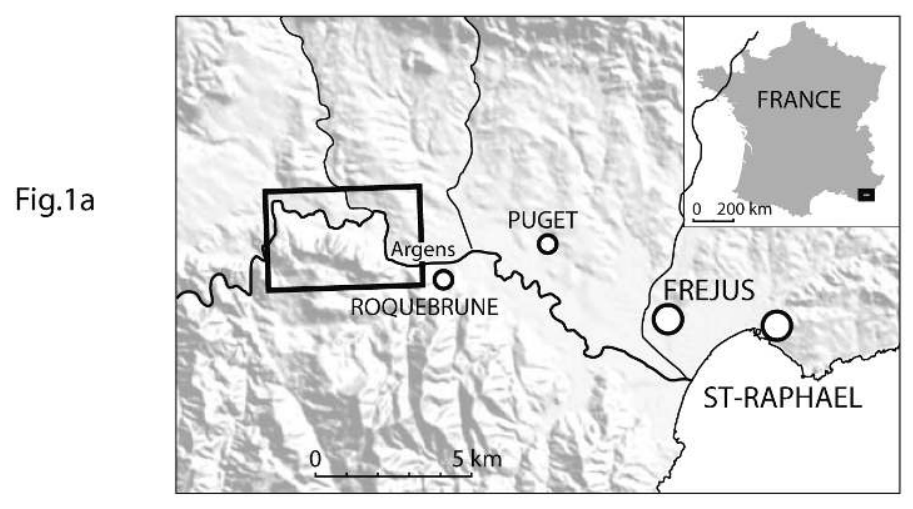

Fig.1b

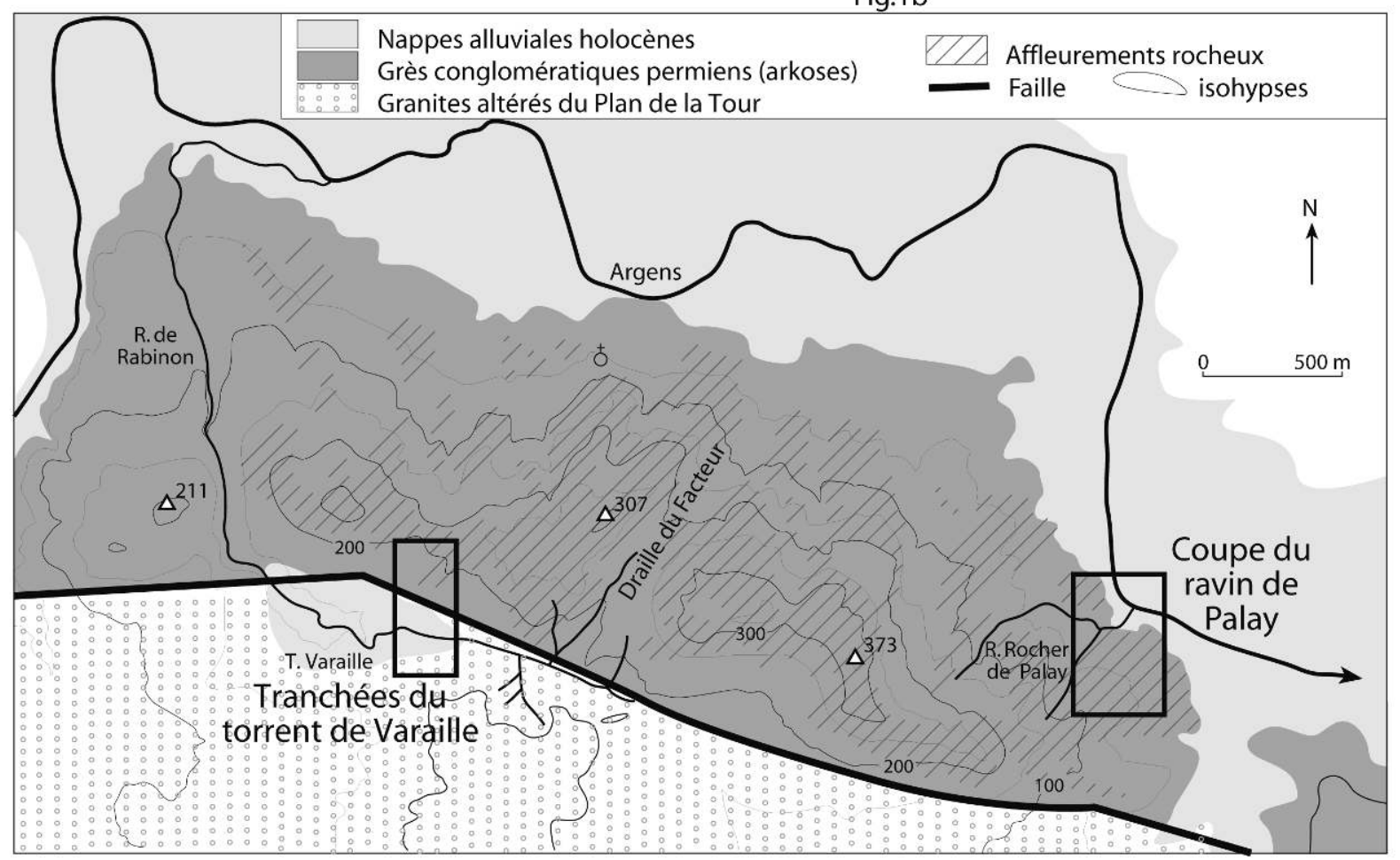

Figure 1a : Croquis de localisation du Rocher de Roquebrune-sur-Argens.

Figure 1a: Location of the rocky hill of Roquebrune-on-Argens.

Figure $1 \mathrm{~b}$ : Contexte géologique des sites.

Figure 1b: Geological context of the sites.

Le secteur du Rocher de Roquebrune se situe quelques kilomètres à l'amont du fond de la ria holocène de l'Argens (maximum d'ingression marine). La prise en compte d'une zone d'étude aussi éloignée du rivage actuel (une dizaine de $\mathrm{km}$ ) pour étudier l'évolution de la basse vallée de l'Argens se justifie par la nécessité de comprendre les dynamiques érosives qui ont contribué au comblement de la ria. Intervenue dans le cadre d'un ralentissement de la remontée du niveau marin à partir de $6000 \mathrm{BP}$ environ (Dubar et Anthony, 1995, Dubar 2004), la progradation du trait de côte est en effet liée aux apports sédimentaires transitant par l'Argens. Issus de l'érosion des couvertures sédimentaires et du socle, que conditionne la lithologie du bassin-versant, ces apports varient en intensité en fonction de deux facteurs, le facteur climatique et le facteur anthropique, par l'intermédiaire notamment de l'activité agricole et des aménagements qu'elle a générés en particulier sur les massifs qui délimitent le bassin-versant. Le Rocher de Roquebrune, dont l'occupation protohistorique et antique est bien connue, offrait la possibilité de confronter les observations géomorpholo- 
giques et archéologiques afin d'appréhender, à l'échelle de ce petit massif, l'histoire des processus érosifs dans le bassinversant de l'Argens.

La recherche a donc porté sur les petits bassin-versants affluents proches du delta, qui offrent la possibilité d'étudier plus précisément la chronologie des épisodes détritiques et de les associer à des forçages anthropiques et/ou climatiques. Les résultats de l'étude géomorphologique réalisée dans les vallons de Varaille et du Rocher de Palay sont présentés ici, puis brièvement comparés avec les données géoarchéologiques acquises sur d'autres sites provençaux, qui avaient permis de montrer l'importance et la variabilité des dynamiques géomorphologiques et des colmatages sédimentaires associés à l'anthropisation (Provansal 1995, Jorda et Provansal 1996, Bruneton 1999).

\section{Le Rocher De RoQuebrune, CONTEXTE STRUCTURAL ET GÉOMORPHOLOGIQUE}

Le Rocher de Roquebrune forme un relief escarpé dominant de $350 \mathrm{~m}$ la rive droite de la basse vallée de l'Argens. Ce petit massif, qui culmine à $373 \mathrm{~m}$, est formé par les arkoses rouges permiennes, qui constituent la base de l'enveloppe sédimentaire du massif cristallin primaire des Maures (Fig. 1b). Les arkoses, grès conglomératiques siliceux très résistants, ont été mises en relief par l'érosion différentielle des roches tendres périphériques (marnes permiennes de la dépression périphérique au nord, granites altérés du Plande-la-Tour au sud). Le massif est caractérisé par un relief tabulaire, déchiqueté par des ravins profonds (jusqu’à plusieurs dizaines de mètres). L'Argens y inscrit à l'ouest des gorges en surimposition. Sur son versant sud, un faisceau de failles E-O, au contact entre les arkoses et les granites altérés, détermine le tracé de petits talwegs d'angle de faille, dont les versants dissymétriques traduisent l'inégale résistance des arkoses et des granites altérés. L'un d'entre eux, le vallon de Varaille, prend sa source en contrebas de l'habitat perché tardo-antique de Sainte-Candie, tandis que ses affluents trouvent leur origine à proximité des sites protohistoriques de "La Croix Bouf » et du "massif de Roquebrune " (Fig. 2). Le vallon de Varaille est perché vers l'ouest au-dessus de la gorge étroite du ravin de Rabinon, à l'amont de laquelle il s'élargit et se colmate. Une tranchée ouverte à la pelle mécanique a permis d'analyser ce remplissage sédimentaire.

Sur le flanc nord du Rocher de Roquebrune, les ravins très abrupts (pentes $>10 \%$ ), inscrits dans les arkoses, descendent directement vers la vallée de l'Argens. Une coupe naturelle à l'aval de l'un d'entre eux (site du Rocher de Palay) a permis d'observer des dépôts torrentiels, interstratifiés avec les apports alluviaux de la basse terrasse de l'Argens. Ces apports torrentiels latéraux sont vraisemblablement à l'origine $\mathrm{du}$ méandrage localisé de ce fleuve.

\section{HistoIRE DE L'OCCUPATION DU SOL}

Les prospections pédestres, sondages (Bertoncello 1995, 1996, 2003) et fouilles (Bertoncello et Codou 2005) effectués sur le Rocher depuis 1995 permettent de dresser un tableau assez précis de l'occupation du massif entre le Néolithique et le haut Moyen Âge.

Les vestiges les plus anciens ont été repérés sur le piémont nord du Rocher : les énormes blocs détachés de la falaise dans le secteur de La Roquette ont servi d'abris sous roche au Néolithique moyen et à l'Âge du Bronze. C'est au cours de la Protohistoire que l'occupation du Rocher semble la plus intense : dix gisements ont livré du mobilier datable de l'Âge du Fer et six autres de la céramique modelée trop peu caractéristique pour proposer une datation précise (Fig. 2). Au sud du Rocher, entre ce dernier et le massif des Maures, les plateaux des Sausses et des Bas Pétignons sont aussi occupés au cours de la période préromaine : deux sites et deux zones d'épandage ont été reconnus en prospection, sans que l'on puisse préciser davantage leur chronologie (Bertoncello 2003). Sur le Rocher, l'occupation se concentre à l'Âge du Fer dans des habitats groupés, fortifiés ou non (Bertoncello 1999, 348-352). L'examen de leur répartition suggère un déplacement du versant sud vers le versant nord. En effet, si les quatre établissements repérés sur le versant nord, qui domine l'Argens, sont tous occupés au $\mathrm{II}^{\mathrm{e}}$ et/ou I $\mathrm{I}^{\mathrm{er}}$ siècle av. J.-C. (Fig. 2 : Rochers 2, Rochers 5, Les hautes Roques 1 et 2 ), les trois habitats groupés situés sur le versant sud, donc du côté du Rabinon, sont probablement antérieurs (fig. 2) . Tel est le cas de l'oppidum du Massif de Roquebrune, le plus vaste $\left(50000 \mathrm{~m}^{2}\right)$, qui a fait l'objet de sondages dans les années soixante (Liégeois et Girard 1992) et a livré un mobilier suffisamment fourni pour être daté du second Âge du Fer, entre le ve et le II siècle av. J.-C.). Surplombant le vallon de Varaille, la petite enceinte $\left(500 \mathrm{~m}^{2}\right)$ de La CroixBœuf a livré quelques fragments d'amphore massaliète qui suggèrent de la dater du début du second Âge du Fer. Enfin, le troisième établissement, La Haute Rouquaire, occupe un petit col barré de chaque côté par un long mur en pierres sèches. En dépit des prospections, les rares tessons de céramique modelée retrouvés sur ce site sont insuffisants pour proposer une datation.

Le Rocher semble inoccupé à l'époque romaine, si l'on excepte la découverte de plusieurs tessons d'amphore africaine associés à quelques fragments de tuiles, pouvant 

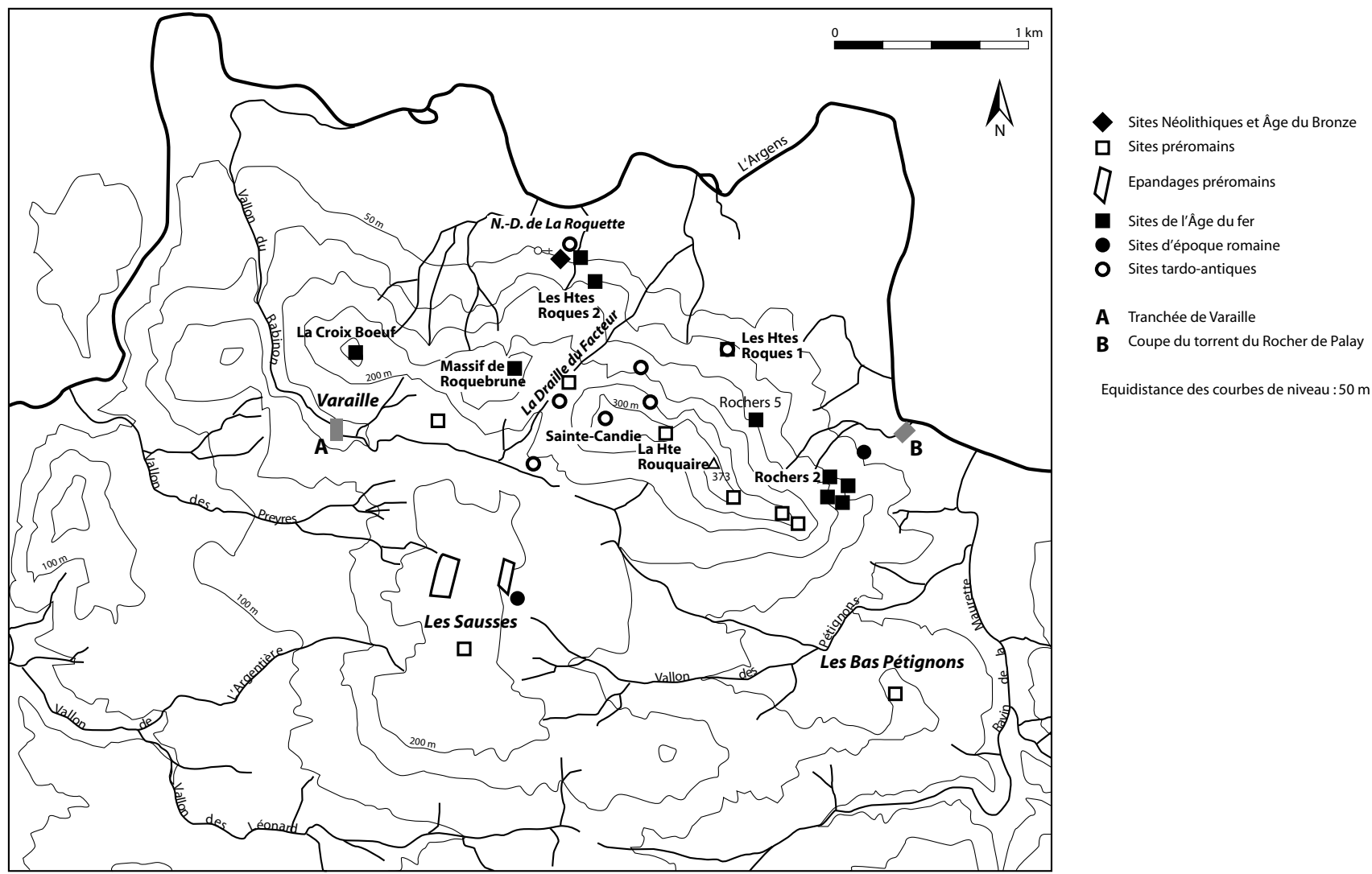

Figure 2 : Carte archéologique du Rocher de Roquebrune, du Néolithique à la fin de l'Antiquité. Figure 2: Archeological map of the rocky hill of Roquebrune-on-Argens, from Neolithic to Late Antiquity.

éventuellement appartenir à une sépulture (?). Un établissement existe en revanche sur le plateau des Sausses au haut Empire.

À la fin de l'Antiquité, la reprise de l'occupation du Rocher se manifeste par la création d'un habitat groupé sur le plateau de Sainte-Candie, qui surplombe la partie amont du vallon de Rabinon (Fig. 2). Véritable village, doté d'une église et d'un rempart enserrant près de 8 ha, cet habitat semble avoir connu une occupation assez brève à la charnière des $\mathrm{V}^{\mathrm{e}}$ et $\mathrm{VI}^{\mathrm{e}}$ siècle, suivie d'une réoccupation - partielle? - dans la première moitié du viII ${ }^{\mathrm{e}}$ siècle (Bertoncello et Codou 2005). L'église a fait l'objet d'une fréquentation plus durable, jusqu'aux XII ${ }^{\mathrm{e}}$-XIII ${ }^{\mathrm{e}}$ siècle au moins. D'autres points d'occupation contemporains de Sainte-Candie ont été repérés sur le Rocher, qu'il s'agisse de cabanes isolées autour du village, ou de la réoccupation de l'habitat de l'Âge du Fer des Hautes Roques 1 (Fig. 2).

\section{MORPHOGENÈSE HOLOCÈNE DU ROCHER DE ROQUEBRUNE}

Elle est décrite par deux ensembles stratigraphiques (sites de Varaille et du Rocher de Palay), dont la chronologie repose sur 4 datations par le ${ }^{14} \mathrm{C}$. Dans les deux cas, les formations sédimentaires reposent directement sur le substrat des arkoses permiennes, ici représentées par un faciès grésoconglomératique altéré.

\subsection{Stratigraphie du site de Varaille}

Situé sur la ligne de faille au sud du Rocher de Roquebrune, ce petit vallon s'évase sur $300 \mathrm{~m}$ de large environ, avant la rupture de pente des gorges qui le sépare de la vallée de l'Argens. Il reçoit de ses deux versants des apports différents par leur dynamique et leur lithologie. Sur sa rive droite, les ravins en pente forte, inscrits dans les grès et conglomérats permiens, génèrent des apports de galets de forte taille. Sur sa rive gauche, en pente douce, le colluvionnement remanie les arènes granitiques altérées. Le ravin actuel est dominé sur sa 


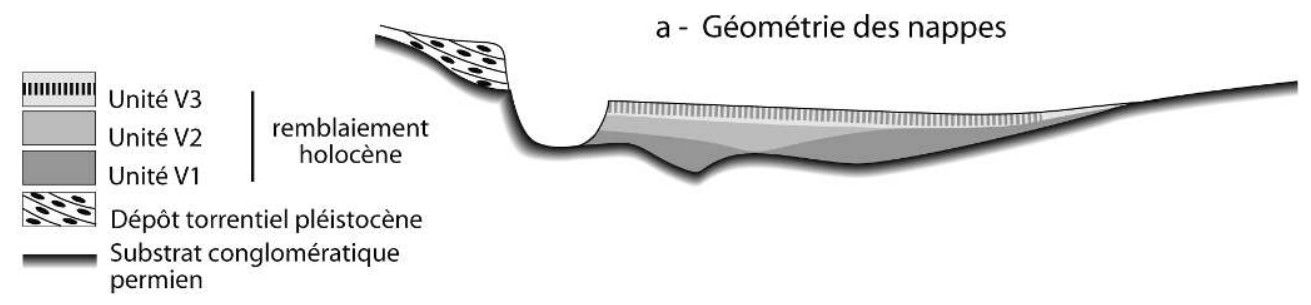

b - Coupes sériées du remplissage sédimentaire

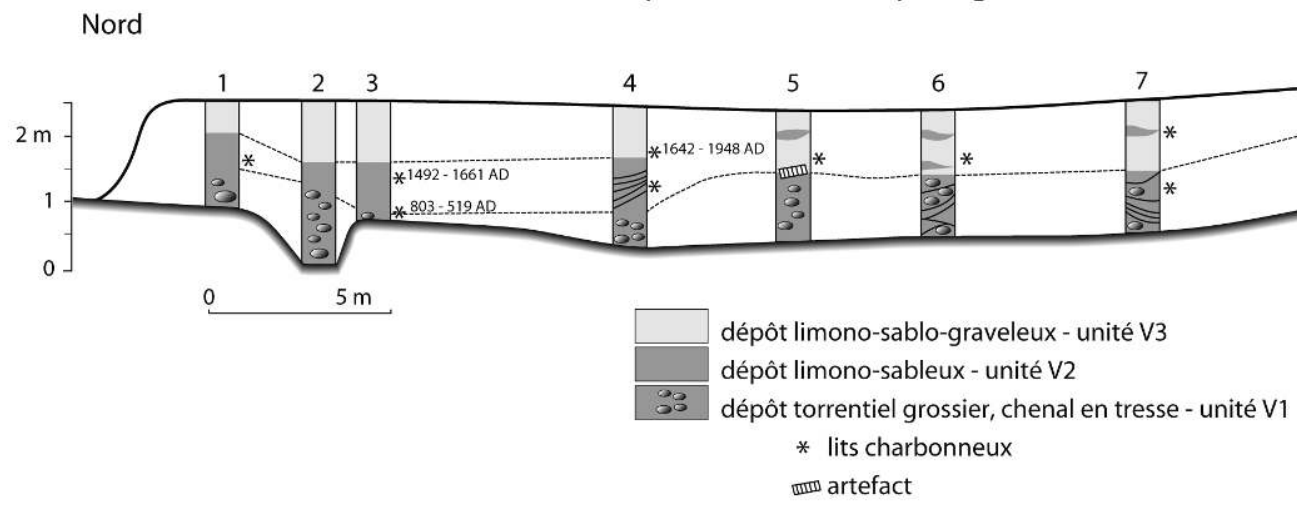

Figure 3 : Stratigraphie du vallon de Varaille.

Figure 3: Stratigrafy of Varaille valley.

rive droite, au nord, par une haute terrasse alluviale de galets $(+10 \mathrm{~m})$, puis par une basse terrasse en rive gauche $(+2-$ $3 \mathrm{~m}$ ), au sud. Cette dernière a été ouverte par une tranchée de $35 \mathrm{~m}$ de long et 1,5 à $2,5 \mathrm{~m}$ de profondeur, perpendiculaire au talweg. Sur toute sa longueur, la base de la tranchée atteint le substrat permien. Celui-ci est recouvert par 1,5 à $2 \mathrm{~m}$ de dépôts meubles, correspondant à trois unités stratigraphiques (Fig. 3).

L'unité inférieure (Unité V1) est un dépôt formé de galets de forte taille (15 à $20 \mathrm{~cm}$ de grand axe), associés à une matrice sablo-graveleuse. L'organisation des éléments (disposés à plat, relevés vers l'aval), le litage subhorizontal ou oblique des sables, la présence de lentilles de sables fins microlités ou de limons, décrivent un dépôt de lit mineur en tresse. Les variations d'épaisseur traduisent la présence de plusieurs chenaux divaguant sur toute la largeur de la tranchée, les moins actifs étant situés sur la bordure sud. La lithologie des galets, très proche de celle du substrat arkosique, montre que les apports proviennent des torrents de rive droite. Tous les indicateurs caractérisent un fonctionnement torrentiel, certainement associé à un dépôt en vrac à la fin de crues brutales : importance relative du ballast $(26$ à $76 \%$ du poids des échantillons), taille des galets, tri (Folk et Ward, 1957) très médiocre $(0,98>\sigma>1,4)$ et grossièreté de la fraction sableuse (grain moyen 0,6 à $0,8 \mathrm{~mm}$ ). La granulométrie s'affine au sommet de cette unité, où les sables moyens deviennent dominants. Quelques charbons de bois ont été prélevés dans les lits sableux sommitaux.

L'unité médiane (Unité V2) ravine partiellement le sommet de l'unité précédente, dans laquelle elle inscrit un paléochenal large d'une quinzaine de mètres, profond d'environ $1 \mathrm{~m}$. Elle est formée de sables grossiers et de graviers lités, associés à quelques lentilles de petits galets ou de limons. Cette structure est caractéristique d'un dépôt par une dynamique alluviale. Le pendage oblique et la structure des sables sur la rive sud du paléo-chenal évoquent la présence d'un banc de méandre. La texture (sables $>70 \%$, ballast environ $25 \%$ ), les indices granulométriques des sables (grain moyen hétérogène entre 0,25 et $0,9 \mathrm{~mm}$, tri moyen avec $\sigma$ égal ou < à 1) correspondent à la charge de fond d'un chenal unique, traduisant des écoulements beaucoup moins compétents que dans l'Unité V1. Bien que les caractères géochimiques des deux unités soient très proches, également caractérisés par les faibles teneurs en carbonate de calcium $\left(\mathrm{CaCO}_{3}<5 \%\right)$ et en matière organique totale $(<1,3 \%)$, il est vraisemblable que les sables et les fines (silts fins et argiles) de l'Unité V2 proviennent en partie des altérites et des sols développés sur les granites du versant de rive gauche. Des charbons de bois ont été prélevés dans les sables fins au sommet de cette unité.

L'unité supérieure (Unité V3), épaisse d'1 m environ, est visible sur toute la longueur de la tranchée et recouvre en 
accordance l'unité précédente. Elle correspond à un dépôt sablo-silto argileux massif (fraction inférieure à $0,04 \mathrm{~mm}$ $>30 \%)$, non stratifié, mal trié $(1,3>\sigma>1,4)$, comprenant quelques micro-lits gravillonnaires sur sa marge sud. Ces caractéristiques correspondent à des écoulements peu compétents, liés vraisemblablement aux débordements du ruisseau lors des crues et/ou à des apports colluviaux à partir du versant de rive gauche. La bioturbation affecte toute l'épaisseur de cette unité, traduisant l'existence d'un faible taux de sédimentation. Des charbons de bois sont disséminés dans toute l'épaisseur du dépôt, à la base duquel un d'entre eux a été prélevé pour datation.

Trois dates 14C obtenues sur charbons de bois permettent de dater les 3 unités de la coupe de Varaille : $2541 \pm 45$ BP (803-519 av. n. è) au sommet de l'Unité V1 (AA-50164); $290 \pm 35$ BP (1492-1661 de. n. è) à la base de l'Unité V2 (Ly 11542) ; $206 \pm 37$ BP (1642-1948 de. n. è) à la base de l'Unité V3 (AA-50163).

La première permet de situer la nappe torrentielle inférieure dans le premier Âge du Fer. La zone-source de l'érosion génératrice de ce dépôt, située dans les arkoses du Rocher de Roquebrune, suggère de mettre en rapport cet épisode avec l'occupation du Rocher, qui est effectivement intense durant la Protohistoire. On constate cependant que l'occupation des sites de «La Croix Bœuf » et du «Massif de Roquebrune » semble légèrement plus récente (début du second Âge du Fer) que la « crise » morphologique. Rappelons toutefois les limites de la datation de ces sites qui, en raison de la rareté du mobilier archéologique, reste imprécise. Quoi qu'il en soit, les facteurs à l'origine de l'érosion, puis du dépôt de ces sédiments sont difficiles à identifier précisément : on doit sans doute voir dans ce fonctionnement torrentiel la coïncidence de facteurs anthropiques, encore mal datés, et d'une période climatique favorable à des écoulements brutaux, très compétents.
Les deux autres datations, cohérentes entre elles, situent les Unités V2 et V3 entre la fin du xv ${ }^{e}$ siècle et la période contemporaine. Il paraît logique de les mettre en relation avec la détérioration climatique plus humide du Petit Âge Glaciaire. L'Unité V2 correspond au remblaiement du talweg de Varaille, qui ne parvient pas à évacuer toute sa charge sédimentaire, alors que l'Unité V3 témoigne du redémarrage de l'incision, puisqu'elle n'est formée que de dépôts de débordement ou d'apports des versants. Cette évolution est comparable à celle de la plupart des cours d'eau en France méditerranéenne depuis la fin du XIX ${ }^{e}$ siècle, caractérisés par une reprise d'incision. Cette dernière est attribuée à la remontée forestière, qui stabilise les versants et réduit leur contribution à la charge solide des rivières (Landon et al., 1994, Liebault et al., 2002).

\subsection{Stratigraphie du site du Rocher de Palay}

Le petit torrent du Rocher de Palay descend directement du point culminant du Rocher de Roquebrune. Il s'écoule sur le substrat arkosique qu'il entaille faiblement; puis il incise à l'aval un cône de déjection épais de plus de $5 \mathrm{~m}$. C'est ce dernier qui a été étudié, grâce à la coupe naturelle liée à l'incision du torrent. Le piémont nord du massif est jalonné de cônes similaires. L'un d'entre eux (cône de la Draille du Facteur, en amont du torrent du Rocher de Palay) a fait l'objet de tranchées ouvertes à la pelle mécanique. Celles-ci n'ont traversé que les limons alluviaux massifs de la terrasse récente de l'Argens, sans possibilité de datation.

Le cône du torrent du Rocher de Palay passe latéralement vers l'aval aux dépôts limoneux de l'Argens, démontrant la simultanéité du dernier épisode détritique sur les versants et dans la plaine.

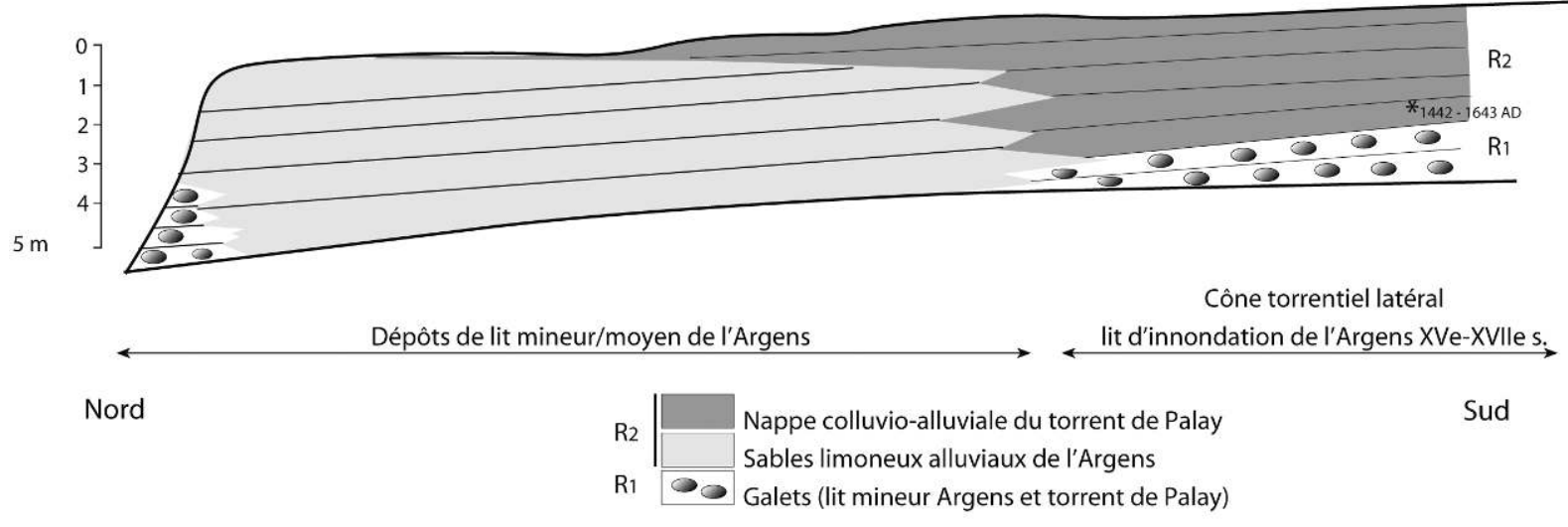

Figure 4 : Stratigraphie du ravin du Rocher de Palay. Figure 4: Stratigrafy of Rocher de Palay ravine. 
Le cône du Rocher de Palay comporte deux unités stratigraphiques superposées (Fig. 4). À la base (Unité R1) se trouve une formation peu épaisse (quelques décimètres) de petits galets (diamètre 2,5 à $5 \mathrm{~cm}$ ), à litage faiblement incliné vers le nord, emballés dans une matrice sablo-limoneuse. La pétrographie et la morphologie de ce matériel montrent qu'il a été apporté à la fois par l'Argens (galets calcaires émoussés) et par le torrent du Rocher de Palay (blocs d'arkose anguleux). Les sables, grossiers, mal triés $(0,8>\sigma>1,3)$ et peu carbonatés $\left(\mathrm{CaCO}_{3}<15 \%\right)$, confirment l'importance des apports du versant.

L'essentiel du cône est formé par un prisme de sédiments fins, de 3 à $5 \mathrm{~m}$ d'épaisseur, présentant un faible pendage vers le nord (Unité R2). Le dépôt est sablo-limoneux à proximité de l'Argens, limono-sableux vers l'amont, bioturbé dans sa partie supérieure. À l'aval, les sables (> $55 \%$ de la texture), fins et assez bien triés $(0,7>\sigma>0,8)$, ont été vraisemblablement déposés par l'Argens; leur proportion diminue en amont $(<30 \%)$ où leur tri se dégrade. Cette organisation traduit l'interstratification d'apports longitudinaux, d'origine alluviale, et latéraux, essentiellement colluviaux. Des charbons de bois ont été prélevés dans la partie médiane de ces derniers.

La date obtenue (357 \pm 40 BP : 1442-1643 de n. è., AA50165) permet de considérer le dépôt de l'Unité R2 comme contemporain de celui de l'Unité V2 et de le situer dans l'épisode climatique du Petit Âge Glaciaire.

\subsection{Interprétation}

Les relevés stratigraphiques réalisés sur les sites de Varaille et du torrent du Rocher de Palay démontrent l'existence d'une « crise " érosive récente, qui pourrait débuter dans la seconde partie du Xve siècle. Elle correspond évidemment à la détérioration climatique humide du Petit Âge Glaciaire, qui redonne aux petits torrents locaux le débit nécessaire pour évacuer les altérites produites sur les versants. L'épuisement de ces stocks détritiques, puis la remontée forestière probable dans la seconde partie du XIX ${ }^{e}$ siècle amènent à une reprise de l'incision sur les deux sites, qui est datée à Varaille entre le XVII et le $\mathrm{XX}^{\mathrm{e}}$ siècle.

Il est également remarquable que la seconde phase importante d'occupation du Rocher de Roquebrune - à la fin de l'Antiquité - n'ait pas laissé de signature paléoenvironnementale perceptible dans le fonctionnement du torrent de Varaille, alors même que le principal point de peuplement à cette époque (entre la fin du ve siècle et le milieu du vi ${ }^{e}$ siècle apr. J.-C.), l'habitat perché de Sainte-Candie, surplombe directement la tête du ruisseau. Loin d'être anecdotique, cette occupation se manifeste par l'installation d'un véritable village, doté d'une église et d'un rempart qui enceint un espace de près de 8 ha. Doit-on alors imputer à son caractère relativement éphémère (la durée exacte d'occupation du site est difficile à mesurer mais n'a pas dû excéder un siècle) ce faible impact sur le milieu, ou au moins son absence d'enregistrement dans la morphogenèse ? Il faut également tenir compte des pratiques agricoles, qui sont, plus que l'habitat, les véritables générateurs de l'érosion. Or nous ignorons, en l'état actuel des recherches à Sainte-Candie, quelles étaient les activités pratiquées par les habitants et la localisation des espaces cultivés. Si l'on considère les conditions topographiques et pédologiques aux abords du site, il n'est pas exclu que les champs se soient trouvés en contrebas du Rocher, sur le Plateau des Sausses par exemple, qui offre des terrains plans et des sols plus profonds. Dans ce cas, ce n'est pas forcément dans le Torrent de Varaille, dont la zone-source est davantage tournée vers le Rocher, que les éventuels effets morphosédimentaires de ces mises en valeur se feraient ressentir.

La faiblesse relative de la morphogenèse au cours des derniers siècles semble paradoxale, étant donné l'importance connue de l'exploitation agricole des terroirs pour cette période. On peut proposer, à titre d'hypothèse, que l'abandon des sites perchés a permis depuis longtemps le reboisement des bassins torrentiels, alors que sont mobilisées désormais d'autres zone-sources (sols, arène granitique) sur les collines de rive gauche du torrent de Varaille.

\section{ConClusion}

Les observations géomorphologiques réalisées sur les piémonts du Rocher de Roquebrune fournissent les premiers jalons pour restituer l'histoire du détritisme dans le bassinversant de l'Argens. Ce genre d'étude doit être multiplié de manière à évaluer la portée géographique, strictement locale ou micro-régionale, des phases détritiques repérées à Roquebrune. Cela permettra de confronter cette histoire des versants aux rythmes de comblement de la ria, dont la chronologie est désormais mieux calée grâce aux recherches en cours.

On peut d'ores et déjà noter que le fonctionnement torrentiel du ruisseau de Varaille se rapproche de celui d'autres sites provençaux du premier Âge du Fer (oppidum de Bramefan, Mouriès, Glanum) et, plus précisément encore de celui de sites varois (Cavalaire, Signes, Porquerolles). Toutefois, si les recherches conduites sur ces sites (Provansal et Morhange 1994, Jorda et Provansal 1996, Provansal 1996, Bruneton 1999) ont montré le rôle moteur joué par l'anthropisation - au travers de l'exploitation des versants notamment dans le déclenchement de processus érosifs (et inversement 
l'absence de déstabilisation des sols dans des secteurs non anthropisés, comme sur les sites des Barres dans les Alpilles ou du Vallat Neuf au bord de l'étang de Berre), les données recueillies à Varaille mettent davantage en évidence la complexité des interactions entre processus anthropiques et climatiques, dont les effets sur les écosystèmes ne peuvent se réduire à des réponses simples et univoques. Entrent certainement en jeu dans ces interactions non seulement les modalités de l'occupation des sols, mais aussi celles de leur mise en valeur agricole (types d'exploitation, intensité, localisation des champs, etc.), dont la réalité archéologique est bien plus difficile à percevoir.

Les relevés stratigraphiques réalisés sur les sites de Varaille et du torrent du Rocher de Palay démontrent l'existence d'une " crise " érosive récente, qui pourrait débuter dans la seconde partie du Xve siècle. Elle correspond évidemment à la détérioration climatique humide du Petit Âge Glaciaire, qui redonne aux petits torrents locaux le débit nécessaire pour évacuer les altérites produites sur les versants. L'épuisement de ces stocks détritiques, puis la remontée forestière probable dans la seconde partie du $\mathrm{XIX}^{\mathrm{e}}$ siècle amènent à une reprise de l'incision sur les deux sites, qui est datée à Varaille entre le $\mathrm{XVII}^{\mathrm{e}}$ et le $\mathrm{XX}^{\mathrm{e}}$ siècle.

Sur le site de Varaille, il est intéressant de souligner que la torrentialité est plus dynamique au premier Âge du Fer qu'au Petit Âge Glaciaire, alors que les indicateurs climatiques démontrent l'importance de ce dernier en Europe Occidentale (Magny, 1995). Outre la présence probable de stocks détritiques hérités encore importants sur les versants, il faut peutêtre y voir surtout la conjonction d'une anthropisation locale importante et d'une recrudescence de pluies efficaces, même si le manque de précision dans la datation des établissements protohistoriques installés sur le versant sud du Rocher ne permet pas d'affiner l'analyse de ces interactions.

\section{Remerciements}

Nous remercions Jacques Bérato (Centre Archéologique du Var) qui a identifié le matériel céramique de l'oppidum du Massif de Roquebrune, et M. Gaston Senes qui nous a autorisé à effectuer une tranchée sur son terrain de Varaille.

\section{Bibliographie}

Bertoncello, F., 1995. Roquebrune-sur-Argens, Rochers, in Bilan Scientifique SRA PACA 1995, Aix-en Provence, Service Régional de l'Archéologie/ministère de la Culture, 1996, p. 231-232.

ArcheoSciences, revue d'archéométrie, 30, 2006, p. 173-180
Bertoncello, F.,1996. Roquebrune-sur-Argens/Le Muy: Les Rochers, in Bilan Scientifique SRA PACA 1996, Aix-en Provence, Service Régional de l'Archéologie/ministère de la Culture, 1997, p. 133-136.

Bertoncello, F., 1999. Le peuplement de la basse vallée de l'Argens de la fin de l'Âge du Fer à la fin de l'Antiquité, Thèse de Doctorat, Aix-en-Provence, 1999, 519 p., 205 fig.

Bertoncello, F., 2003. Roquebrune-sur-Argens : Rocher de Roquebrune, in Bilan Scientifique SRA PACA 2003, Aix-en Provence, Service Régional de l'Archéologie, Ministère de la Culture, 2004, p. 189-190.

Bertoncello, F. et Codou, Y., 2005. Les fouilles de l'habitat perché de Sainte-Candie à Roquebrune-sur-Argens (Var) : premiers résultats, in X. Delestre, P. Périn (dir.), La Méditerranée et le monde mérovingien: témoins archéologiques. Actes des XXIII ${ }^{\mathrm{e}}$ Journées internationales d'archéologie mérovingienne, Arles, 11-13 oct. 2002. Aix-en-Provence, 2005 (Bulletin Archéologique de Provence, suppl. 3), p. 135-142.

Bruneton, H., 1999. Évolution holocène d'un hydrosystème nordméditerrranéen et de son environnement géomorphologique, les plaines d'Arles, thèse Univ. de Provence, Aix, 360 p.

Dubar, M. 2004. L'édification de la plaine deltaïque du Bas Argens (Var, France) durant la Protohistoire et l'Antiquité. Application d'un modèle numérique $2 \mathrm{D}$ à l'archéologie, Méditerranée, 2004, 1-2, p. 47-54.

Dubar, M. et Anthony, E., 1995. Holocene environmental change and river-mouth sedimentation in the Baie des Anges, French riviera, Quaternary Research, 1995, 43, p. 329-343.

Landon, N. et Piégay, H., 1994. L'incision de deux affluents subméditerranéens du Rhône : la Drôme et l'Ardèche, Revue de Géographie de Lyon, 1, p. 63-72.

Liébault, F. et Piégay, H., 2002. Causes of 20th century channel narrowing in mountain and piedmont rivers of southeastern France, Earth Surface Processes and Landforms, 27, p. $425-444$.

Jorda, M. et Provansal, M., 1996. Impacts de l'anthropisation sur le détritisme en France du SE (Alpes du Sud et Provence), Bull. Soc. Géol. Fr., t. 167, n 1, 1996, p. 159-168.

Liégeois, J. et Girard G., 1992. Découverte d'un camp de l'Age du Fer à Roquebrune-sur-Argens (Var), Chroniques de SantaCandie, 43, 1992, p. 7-14.

MAGNY, M., 1995. Une histoire du climat, des derniers mammouths au siècle de l'automobile, Paris, Errance, 175 p.

Provansal, M., 1996. The part of climate in morphogenesis from the Bronze Age in Provence, southearn France, The Holocene, 5, 3, 1996, p. 348-353.

Provansal, M. et Morhange, C., 1994. Seuils climatiques et réponses morphogéniques en Basse Provence depuis 5000 ans, Quaternaire, 5, 3-4, 1994, p. 113-118. 